\title{
Bicanoid- Smart Tag for Object Tracking
}

\author{
Ketki Pendse, Manasi Deshpande , \\ Saee Deshpande, Samruddhi Chavan \\ Prof. Suhas Chavan \\ Assistant Professor \\ Information Technology Department, \\ RMD Sinhgad School of Engineering, Pune
}

\begin{abstract}
Object Tracking is one of the most significant and challengingtasks of smart video surveillance system. It gains increasing attention due toits academic and commercial potential. The ability to track objects has improved drastically duringthe last decades; however, it is still considered a complex problem tosolve.

Misplacing things in house is always frustrating and timeconsuming experience when they are of utmost need. Every house has a history of searching for keys, pen-drives, wallets and hand-purses and it is a difficult job. So there is need for the system that can help out with this problem.

This paper gives a detailed review of the different object tracking methods used by the researchers.The goal of this paper is to analyse, examine, evaluate, and make a summary of various approaches towards object tracking.
\end{abstract}

\section{Keywords-Object Tracking, Bicanoid, Smart Tag, Bluetooth} Low Energy (BLE), Smartphone.

\section{INTRODUCTION}

Object tracking is an area within computer vision which has many practical applications such as video surveillance, sports analysis, human-computer interaction, finding lost objects and robot navigation [1]. It isa well-studied problem, and in many cases a complex problem to solve. Tracking systems are designed to monitor devices or persons, have become prevalent issues in modern technology. The manual manners of locating equipment are rigorous and unproductive.

People losing their belongings like keys, pen-drives, wallets and hand-purses have become a common issue. Moreover the belongings are lost mostly due to misplacement. It takes to much of effort to go in search of the lost belongings and even results in waste of time especially, when they are of badly needed.

People have many ways of solving the problem of finding their lost objects. One of the many ways is to place the important object at pre-specified, consistent location after using it. This avoids the problem of losing those objects. But it does not helpthe user if the object has not been placed where it "should"have been placed.

Some of the existing systems are uses GPS, GSM, RFID and Bluetooth signal strength implementation techniques for object tracking.

- $\quad$ RFID [2] [3] [5] uses either passive or active tags to track objects. Though RFID tags are very cheap, small and suitable for tracking objects, the sensors are considerably more expensive and require high configuration and software installations. Also RFID signals are easily blocked by Objects and other radio waves
- Global Positioning System (GPS)[4][5]is also used for findingobjects that has been misplaced in a shortperiod oftime without much effort. But the existing indoor localization is not accurate due to the poor signal quality of the GPS.

Object Tracking System is used to find our belonging been misplaced in a short period of time without much effort. To solve the issues with above technology used, the proposed application will help to track the lost object using Bluetooth low energy device that gives high signal strength measurement and link quality. A mobile application is created that can be used only on smart phones, as an interface between the wireless sensors and the user. The transmitter end sends a signal to the receiver sensor, which after being traced will start giving buzzer if the object is in a short range from the user in order to notify the user regarding the lost item

\section{LITERATURE SURVEY}

Robert J. et al.[6] implemented frequently Lost Objects (FLO) system for finding frequently lost personal objects such as wallets, cell phones, and sunglasses using indoor position sensing and spatialized audio. This system uses small radio-frequency tagsattached to each object.User would like to track anindoor positioning system to perform the tracking of theseobjects. The interaction between system and user is carried out via LCD touchpanels placed strategically throughout the house. System guides the user to the lost object using buzzer.

GPS-GSM system in [4] helps to navigate and locate the lost item if it is out of a specified range. The user has to select an item from the list of items in the mobile application to which the sensors are attached. Pseudo codes are sent in a random manner to all the sensors in the form of radio waves. These codes are received by the sensors placed in the items. When receiver and code matches ,alarm system or the item will starts ringing.

Author of [5] implemented a GPS and Bluetooth Based Object Tracking System. The system makes use of Global Positioning System (GPS) [4] for tracking long range objects and Bluetooth technology for short range objects. The system allows a user to view the present position of the targetobject on Google Map through an android application. Hence each target object consists of tags that have both GPS and Bluetooth module. The system can also help to find the objects lost through robbery within a short span of time.

Julie A et al. [7] provide a solution for finding lost items for the visually impaired people. The paper gives evaluation of 
a mobile solution, called FETCH, for allowing the visually impaired to track and locate objects they lose frequently. The devices owned by the users such as their cell phone or laptop are used by FETCH, to locate objects .

Chiao-Wen Kao et al.[8] proposes a method to explore the user's navigation foci and visual tracks by estimating gaze points and mapping them to the objects of video content. The system can track the target objects efficiently even when the target objects are lost. The proposed system can continuously applies sequence scheme and mean scheme throughout to track objectswith higher robustness while they are lost.

GalandaruSwalaganata et al.[9] proposedCamshift method for better and faster tracking result. Camshift method is used as the main tracking technique and Kalman filter is used for prediction and correction. The computational complexity and large memory requirements for the implementation of tracking were reduced with the use this system.

S.M. Kolekar et al.[10] proposed Tag droid" "Tile" for finding lost objects. By attaching the tile on any device like, we may lose like keys, laptops.etc .The app remembers where it previously saw the tile .By sharing with the community of tile lost item can find. One of the methods for finding objects in the home is through the use of computer vision and computational perception is explained in [11]. The system uses cameras to track when objects of interest are moved within a space and can thus report the current location of any object within its field of view.

Ma and Paradiso [12] present a solution that uses a flashlight to activate tags on objects around an environment, causing an LED on the tag to glow. While technologically novel, the current version is not well suited for users with visual impairments.

Arghya P. SaikiaThengal et al.[13] developed a portable battery-operated object tracking system. It is cost-effective and simple-to-use real-time object tracking system using offthe-shelf components. The GPS receiverwill gather its geographical location periodically through thesatellite tracking system. The GSM modem to transmit relevantinformation to some central server at fixed intervals of time, orsend some warning message if it detects some anomaly.

Singh et al. in [14] observed that various object sensingtechnologies suffer from inaccuracies based on environmentalconditions, and proposed an improved Kalman filter that canfilter out the noise and improve tracking accuracy.

Denaxas et al. [15] used GPS to estimate in real time theaverage speed of traffic on every section of urban roads in atown. The approach uses a large and sparse model of the roadsystem, the bus routes, and the fleet dynamics together with a fastsolver that renders the model solution.

Moloo et al. [16] presented a scheme for tracking mobilephone users using GPS and GPRS on mobile phone devicesand raises alerts if the user enters any restricted zone. The restricted zones can be configured dynamically based on therequirements.

\section{PROPOSED SYSTEM}

In today's busy world many people has the annoying habit of losing things or misplacing them. Losing of the objects like keys, pen-drives, wallets and hand-purses have become a common issue. It takes a lot of effort to search the lost belongings and even results in waste of time particularly, when they are of utmost need.

Hence tracking of objects using the Bluetooth system is best for that purpose. With Beacons, we can able to speedily and precisely locate assets devoid of having to search for it everywhere. It is very useful for decreasing over-purchasing tools and rental costs.

The proposed application will help to solve this issue with fewer efforts by using BLE (Bluetooth Low Energy) tags. This application helps to track the BLE tags and estimate the device is near to the mobile by giving buzzer sound.

User needs to add BLE tag to the object. If object is lost then user has to turn ON the BLE frequency by using smartphone. The application starts to track the BLE tags and estimate the device is near to the mobile and provide alert by the BLE device in the form of playing Buzzer.

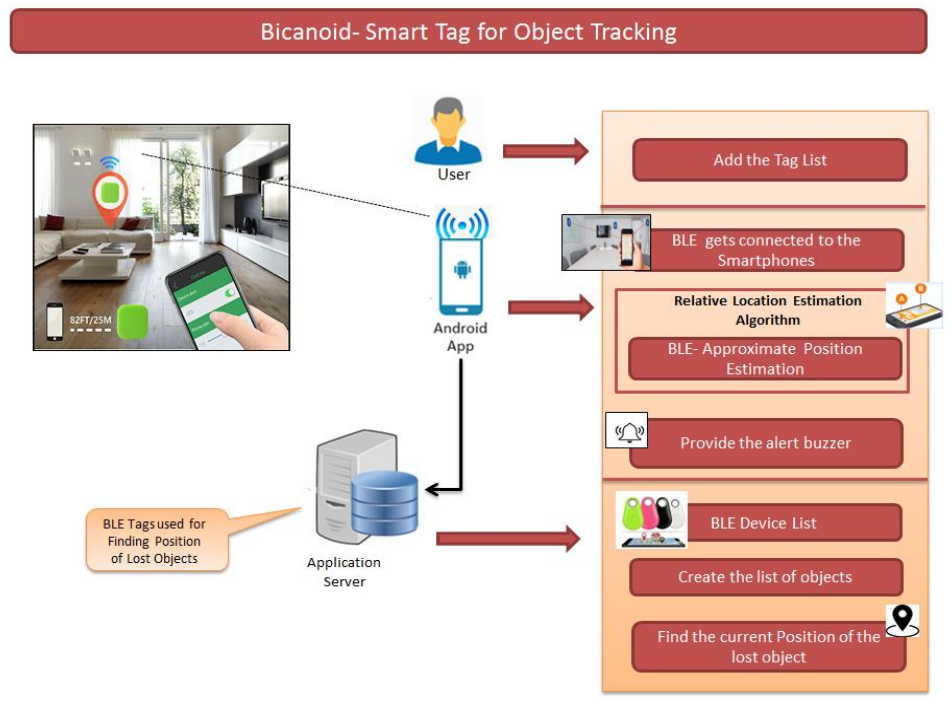

Figure 1: System Architecture 


\section{ADVANTAGES:}

- It requires low energy to Access.

- It is used for the Indoor Application.

- It works without Internet.

- Devices have long battery life.

\section{CONCLUSION}

There is a need of being able to keep track of important assets in indoor environments. As people losing their belongings like keys, pen-drives, wallets and hand-purses have become a common issue. Again the belongings are lost mostly due to misplacement. So there is need for the system or application that can locates lost assets in a cheap and efficient way.

This paper gives the survey of different techniques used for tracking the objects and abstract view of the system that we are going the implement to solve the issued related to the existing system.

We are implementing system with the use of BLE beacons which can be described as small devices broadcasting their identities using Bluetooth Low Energy. The system would prove to be efficient to find the items that are misplaced.

\section{FUTURE WORK}

$>$ In this paper, proposed algorithm is for indoor positioning. The distance between mobile devices and beacon is then calculated using python. Finally, beacons location is determined by trilateration.

$>$ To enhance its performance and functionalities, the system serves as a basis for implementing tracking system that has the following characteristics. System does not require line-of-sight operation.

$>$ The tracker system and the Access Point system implementations require less memory and computational overload.

$>$ In future the boundary for the distance between smartphone (tracking device) and the beacon could be extended.

\section{REFERENCES}

[1] JaeyongJu, Daehun Kim, Bonhwa Ku, HanseokKo,David K. Han,"Online Multi-Object Tracking based on Hierarchical Association Framework",IEEE.

[2] Lun-Chi Chen,Ruey-Kai Sheu, Hui-Chieh Lu, Winston Lo,"Object Finding System Based on RFID Technology",from book Advanced Web and Network Technologies, and Applications: APWeb 2006 International Workshops: XRA IWSN, MEGA, and ICSE, Harbin, China, January 16-18, 2006 Proceedings (pp.383-396).

[3] Rupen Paul V1, Adithi Reddy2, Sujith PS3, Aneesh M,"GPS and Bluetooth Based Object Tracking System", International Journal of Advanced Research in Computer and Communication EngineeringVol. 5, Issue 1, January 2016

[4] Chiao-Wen Kao ; Bor-Jiunn Hwang ; Chaur-Heh Hsieh ; YunTing Huang ; Hui-Hui Chen ; Shyi-Huey Wu ,"The integrated gaze and object tracking techniques to explore the user's navigation", 2014 International Conference on Machine Learning and Cybernetics.

[5] GalandaruSwalaganata ;Muniri ; YessiAffriyenni ,"Moving object tracking using hybrid method", 2018 International Conference on Information and Communications Technology (ICOIACT).

[6] S.M. Kolekar, Saurabh N. Funne,Niranjan M. Tade,Omkar A Rajgire,Ganesh R.Ghorpade,"Tracking System Using Bluetooth Tags and Android app-Tagdroid", IJRERDInternational Journal of Recent Engineering Research and Development (IJRERD)Volume No. 02 -Issue No. 05 , ISSN: 2455-8761 www.ijrerd.com, PP. 81-85.

[7] Nelson, Randal and Green, Isaac, "Tracking Objects Using Recognition", in International Conference on Pattern Recognition (ICPR02), Quebec City, Quebec, August 2002, Volume 2, 1025-1039.

[8] Arghya P. SaikiaThengal ; NamanRastogi ; AbhilashMedhi ; RituranjanSrivastava ; KamalikaDatta ,"Parameter sensing and object tracking using global positioning system", 2016 Sixth International Symposium on Embedded Computing and System Design (ISED).

[9] A. Singh and Sonal.An improvement over Kalman filter for GPS track-ing. InIntl. Conf. on Computing for Sustainable Global Development,pages 923-927, 2016.

[10] E. Denaxas, S. Mpollas, D. Vitsios, and C. Zolotas.Real-time urbantraffic information extraction from GPS tracking on a bus fleet.InIEEESymp.on Computational Intelligence in Vehicles and TransportationSystems, pages 58-63, 2013.

[11] K. Moloo and V. K. Digumber.Low-cost mobile GPS tracking solu-tion.InIntl. Conf. on Business Computing and Globa Informatization,pages 516-519, 2011. 